\title{
Reflexiones sobre Calentamiento Global y Turismo. Principales riesgos ambientales y regiones turísticas afectadas
}

\author{
Reflections on Global Warming and Tourism. \\ Main environmental risks and affected tourist regions
}

\author{
Noelia Aymara Padilla \\ Doctora, Centro de Investigaciones Económicas y Sociales. Universidad Nacional de Mar del Plata, Argentina. \\ Noeliaaymarapadilla@gmail.com | 0000-0003-1233-4803
}

Para citar este artículo: Padilla, N. A. (2020). Reflexiones sobre Calentamiento Global y Turismo. Principales riesgos ambientales y regiones turísticas afectadas. Entorno Geográfico, (20), 1-22. doi: 10.25100/eg.v0i20.10862

\section{Resumen}

Los efectos ambientales relacionados al calentamiento global ponen en riesgo el desarrollo turístico y la estabilidad económica de varios países con una marcada dependencia hacia la actividad. Bajo conceptos provenientes de la Teoría Social del Riesgo y el Binomio ClimaTurismo, se realiza un análisis de los efectos ambientales del Calentamiento Global, tales como, el derretimiento de las nieves eternas, el aumento del nivel del mar, los cambios en las corrientes marinas y los fenómenos meteorológicos extremos (huracanes, inundaciones, precipitaciones, sequias, olas de calor) que afectan al turismo directa e indirectamente. Teniendo en cuenta las regiones de la Organización Mundial del Turismo (OMT), se especificaron casos emblemáticos cuya selección responde al papel del turismo en el desarrollo económica del lugar o a su riqueza en materia de atractivos turísticos. Los resultados permiten dar cuenta de la modificación de situaciones climáticas que favorecen al turismo, la mayor ocurrencia de desastres naturales, la presencia de atractivos en riesgo, la destrucción de infraestructura básica, entre otras cuestiones. Por ello, cobra importancia planificar la actividad turística teniendo en cuenta las amenazas y riesgos derivadas de un aumento de la temperatura atmosférica.

Palabras clave: Calentamiento Global; Turismo; Regiones Turísticas; Riesgos; Vulnerabilidad

\begin{abstract}
The environmental effects related to global warming jeopardize tourism activity and the economic stability of several countries with a marked dependence on activity. Concepts from Social Theory of Risk and the ClimateTourism Binomial are used, and an analysis is made of the environmental effects of Global Warming, such as the melting of the eternal snows, the rise in sea level, the changes in marine currents and the extreme
\end{abstract}


weather events (hurricanes, floods, rainfall, drought, heat waves) that affect tourism directly and indirectly. Taking into account the World Tourism Organization (UNWTO) regions, emblematic cases were identified whose selection responds to the role of tourism in the economic development of the place or its wealth in terms of tourist attractions. The results allow to account for the modification of

Recibido: 30 de diciembre de 2019

\section{Introducción}

El calentamiento atmosférico puede generar un cambio climático global, a partir de una serie de modificaciones físico/ambientales tales como, el derretimiento de las nieves eternas, el aumento del nivel del mar, los cambios en las corrientes marinas y los fenómenos meteorológicos extremos (huracanes, inundaciones, precipitaciones, sequias, olas de calor). El Grupo Intergubernamental de Expertos sobre el Cambio Climático (IPCC) (1990), define al Cambio Climático como la variación estadísticamente significante de un parámetro climático medio o su variabilidad, persistiendo en un período extenso (típicamente en décadas o por más tiempo).

Los efectos ambientales relacionados al calentamiento global afectan al turismo directa e indirectamente y ponen en riesgo la estabilidad económica de varios países con una marcada dependencia hacia la actividad. El turismo es altamente vulnerable al cambio climático ya que el clima es un factor con gran influencia en el climatic situations that contribute tourism, the greater occurrence of natural disasters, the presence of attractions at risk, the destruction of basic infrastructure, among other issues. Therefore, it is important to plan the tourism activity taking into account the threats and risks arising from an increase in atmospheric temperature.

Keywords: Global Warming; Tourism; Tourist Regions; Risks; Vulnerability

\section{Aceptado: 16 de mayo de 2020}

surgimiento de temporadas turísticas o en la elección de los destinos (Organización Mundial del Turismo -OMT, 2007). En este sentido, resulta de interés comprender cuáles son las regiones y los destinos más amenazadas por el calentamiento global y cuáles son las principales consecuencias ambientales.

Con base en ello, el objetivo de este artículo de investigación es reflexionar sobre la relación entre el calentamiento global y el turismo, presentando las principales peligros y riesgos ambientales del calentamiento global, y los destinos turísticos más vulnerables. Para ello, se parte de considerar la propuesta de regionalización turística de la Organización Mundial del Turismo (OMT) y conceptos provenientes de la Teoría Social del Riesgo y del Binomio Clima-Turismo. La temática cobra interés dentro de la Geografía del Turismo, una rama de la Geografía que vincula el turismo con el territorio. La aceptación del carácter espacial del turismo con sus implicaciones a diferentes escalas: local, regional, nacional e internacional, ha 
acrecentado la importancia sobre los análisis territoriales (Gómez Piñiero, 2004). Ello implica contemplar los componentes ambientales que afectan e indicen en el turismo.

\section{Marco teórico}

El clima, según Strahler y Strahler (2000), es una condición característica de la atmosfera próxima a la superficie terrestre, en un lugar o sobre una región determinada. Comprende un conjunto de condiciones atmosféricas medias, y es producto de la combinación de tres elementos: la temperatura, la presión y la humedad. Por su parte, Cuadrat y Pita (1997, p. 9) mencionan: “...El clima sería, pues, lo permanente, lo habitual, lo característico de la atmósfera sobre un lugar, en suma, aquellas condiciones atmosféricas susceptibles, por su permanencia de generar un medio propio" ... que mínimo se puede establecer en treinta años. Frente a esta noción, lo efímero, lo coyuntural, lo fugaz de la atmosfera sería el tiempo, cuyos autores explican que es definido clásicamente como el conjunto de valores que en un momento dado y en el lugar determinado caracterizan el estado atmosférico.

La temperatura atmosférica está regulada en gran parte por el Efecto Invernadero, un fenómeno natural que genera calentamiento atmosférico. Los llamados "Gases del Efecto Invernadero" (GEI) que se encuentran entre los gases atmosféricos, retienen parte de la energía de onda larga reemitida por la tierra..." tienen la propiedad de absorber la radiación en las longitudes de onda de la ventana de radiación. $\mathrm{Su}$ presencia disminuye la emisión desde la superficie terrestre hacia el exterior" ... (Barros, 2005, p. 35).

Tal como explican Barry y Chorley (2003), al calentarse la superficie de la Tierra transforma la luz solar, de alta energía y de longitud de onda corta, en radiación de ondas larga o infrarroja que es reflejada nuevamente hacia la atmósfera. Esa energía puede ser absorbida de manera muy eficiente por algunos de los gases atmosféricos, de manera particular el Dióxido de Carbono (CO2), pero también el vapor de agua, el metano, entre otros. Esto explica que la temperatura más alta de la Tropósfera (capa inferior de la atmósfera) sea justamente el punto de contacto con la superficie del planeta y descienda en altura. Sin el Efecto Invernadero la superficie de la tierra estaría congelada, con una temperatura media global de $-15^{\circ} \mathrm{C}$ aproximadamente.

El calentamiento global puede ser comprendido como un incremento de la temperatura media de la atmósfera asociado a una intensificación de los GEI y una mayor retención de la energía de onda larga. Ciertas actividades humanas generan una mayor concentración de los gases que forman el efecto invernadero, trayendo aparejado una reducción de la energía reemitida hacia el espacio, y un incremento de la temperatura en la tierra que lleva a la alteración de los patrones climatológicos normales en distintas regiones del mundo (Padilla, 2013). En este sentido, el Panel Intergubernamental sobre Cambio Climático (PICC) en su Quinto Informe de 
Evaluación (AR5) del 2014 explica que existe una clara influencia humana en el clima y es extremadamente probable haya sido la causa dominante del calentamiento observado desde 1950 y del aumento de la concentración de gases de efecto invernadero en la atmósfera.

Tal como menciona Barros (2003, p. 6) “... las emisiones de dióxido de carbono, originadas en la combustión de hidrocarburos fósiles, tuvieron un crecimiento de tipo exponencial desde el comienzo del período industrial, y a las mismas se deben sumar las causadas por la deforestación, que son actualmente tres o cuatro veces menores que las primeras" ... El autor explica que prácticamente la mitad del carbono se está acumulando en la atmósfera, con un incremento de las concentraciones de alrededor del $30 \%$ en los últimos 150 años. En el mismo período, la concentración del metano en la atmósfera aumentó un $150 \%$ y la del óxido nitroso un $16 \%$.

El calentamiento atmosférico puede derivar en un cambio climático global que se refiere a cualquier modificación en el clima de una región a través del tiempo, debido a la variabilidad natural o como resultado de las actividades humanas (Houghton et al., 2001). Generalmente se establece a partir de un análisis de datos en un período de tiempo de 30 a 50 años.

En este artículo se abordan, para una mayor compresión de los efectos del calentamiento global, conceptos provenientes de la Teoría Social del Riesgo, tales como peligrosidad, vulnerabilidad, riesgo y desastre. Uno de los autores de mayor reconocimiento en la definición de estos conceptos es Cardona (1993) quien establece que el riesgo ...es la probabilidad de que se produzcan ciertas consecuencias a raíz de un evento natural o provocado, dónde las consecuencias son factor tanto del grado de exposición de los elementos, como de la susceptibilidad de los mismos frente a eventos peligrosos (Cardona, 1993, p. 56). “...El riesgo puede reducirse si se entiende como el resultado de relacionar la amenaza o probabilidad de ocurrencia de un evento, con la vulnerabilidad o susceptibilidad de los elementos expuestos..." (Cardona 1993, p. 72). Bennett y Doyle (1997) definen la fórmula Peligrosidad X Vulnerabilidad= Riesgo, y señalan que la evaluación del riesgo involucra la descripción de las características del peligro y la exposición de la población al mismo.

Así también, Cardona (1993) define a la peligrosidad (o amenaza) como la probabilidad de ocurrencia de un fenómeno físico, tanto natural como tecnológico, que puede presentarse en un sitio específico y en un tiempo dado, produciendo efectos adversos en personas, bienes o su medioambiente. En este sentido, la vulnerabilidad, corresponde a la predisposición intrínseca de un sujeto o elemento a sufrir daño debido a posibles acciones externas (Cardona, 1993, p. 51). La vulnerabilidad también es definida por Blaikie, Cannon, David y Wisner (1996) como las características de una persona o grupo en base a su capacidad para anticipar, sobrevivir, resistir y recuperarse del impacto de una amenaza natural. 
Finalmente, Wilches-Chaux (1993) asocia este término a una incapacidad de la comunidad para absorber los efectos de determinado cambio en su ambiente. Para este autor, la vulnerabilidad como un sistema dinámico, surge de la interacción de una serie de factores y características internas y externas y la redefine como vulnerabilidad global. Dentro de ella, incorpora a las vulnerabilidades social, cultural, política, económica, física, entre otras. La vulnerabilidad física, de importancia para este trabajo, considera la localización de los asentamientos humanos en zonas con altos indicadores de riesgo y a la deficiencia de su estructura física para absorber las acciones de los eventos riesgosos (Wilches-Chaux, 1993). Por último, el término desastre es entendido como “...un evento o suceso que ocurre, en la mayoría de los casos, en forma repentina e inesperada, causando sobre los elementos sometidos alteraciones intensas, representadas en la pérdida de vida y salud de la población, la destrucción o pérdida de los bienes de una colectividad y/o daños severos sobre el ambiente..." (Cardona 1993, p. 45).

La actividad turística es altamente vulnerable al cambio climático. Los destinos insulares, costeros y de montaña son especialmente sensibles a los cambios ambientales provocados por el clima, pues definen nichos de mercado turístico que giran en torno a la naturaleza (OMT, 2007). Estos tipos de atractivos forman parte integral de la oferta turística de muchos países y son muy vulnerables a aspectos como las variaciones en la disponibilidad de agua, la pérdida de biodiversidad, la degradación del paisaje, las alteraciones en la producción agrícola, la erosión e inundación de las zonas costeras, los daños en la infraestructura y una mayor incidencia de enfermedades transmitidas por vectores. Desde la Geografía del Turismo, tal como menciona Gómez Martin (2005), existe una relación bidireccional entre los elementos atmosféricos y las actividades turísticas. La autora menciona que el clima puede constituirse en:

Soporte y factor de localización turística: el clima es uno de los elementos geofísicos que componen el espacio geográfico., crea condiciones ambientales que hacen posible, dificultan o impiden el asentamiento humano. El turismo también se rige por estos imperativos: el clima se convierte en factor de localización turística en el momento en que interviene en el proceso de funcionalización de un territorio.

Recurso turístico: el clima es un recurso turístico natural ya que es un elemento que, por intermedio de la actividad del hombre y de los medios con que cuenta, hace posible la actividad turística y satisface las necesidades de la demanda. Se convierte en recurso en el momento en que es incorporado a un bien o servicio turístico, siendo promovidos para el consumo y utilizados con fines mercantiles a través de cualquier canal de comunicación y comercialización. 
> Factor de atracción turística: los elementos atmosféricos se convierten en factores de atracción turística cuando, fruto de influencias internas (percepción, motivaciones, aprendizaje, personalidad, actitudes) y externas (aspectos económicos, sociales y culturales, clase social, decisiones familiares) adquieren más importancia que el resto de elementos del producto y son valorados positivamente por el turista hasta influir en la decisión de compra.

La relación del turismo con el clima incluye el estudio y elaboración de índices climáticos a partir de variables como la temperatura, las precipitaciones, los vientos, entre otros (Mieczkowski, 1985; Amelung y Viner, 2006). Según Andrade (1998), el clima debe ser considerado como un factor fundamental para la competitividad de los destinos turísticos ya que influye en la estacionalidad y en la continuidad y la regularidad del flujo turístico. Para Hamilton y Lau (2004) el clima es un factor decisivo en la elección de un destino turístico. Por lo cual, las modificaciones climáticas impactan en la atractividad del destino turístico. Para los autores los cambios climáticos no modifican las preferencias de los turistas, pero si los destinos.

Según datos de la OMT (2018), las llegadas de turistas internacionales subieron un $6 \%$ en 2018, claramente por encima del crecimiento del $3.7 \%$ de la economía mundial. El crecimiento del turismo en los últimos años confirma que el sector es hoy uno de los motores más poderosos de crecimiento y desarrollo económico a nivel global. A nivel regional se corroboran los siguientes resultados relacionados al turismo internacional (OMT, 2018):

Las Américas: aumento del $+3 \%$, con resultados mixtos en todos los destinos. El crecimiento fue liderado por América del Norte $(+4 \%)$ y seguido por América del Sur (+3\%), mientras que América Central y el Caribe (ambos -2\%) obtuvieron resultados muy variados, este último reflejando el impacto de los huracanes de septiembre de 2017 Irma y María. > Europa: aumentó un $6 \%$ en comparación con el 2017. El crecimiento fue impulsado por la Europa meridional y mediterránea $(+7 \%)$, Europa central y oriental $(+6 \%)$ y Europa occidental $(+6 \%)$. Los resultados en el norte mostraron escaso crecimiento debido a la debilidad de las llegadas al Reino Unido.

> África: aumento del 7\% en 2018 (África del Norte en $+10 \%$ y subsahariana $+6 \%$ ).

$>$ Oriente Medio: crecimiento del $10 \%$, consolidando su recuperación de 2017.

Asia y el Pacífico: crecimiento del 6\% en 2018. Las llegadas al sudeste asiático aumentaron un $7 \%$, seguidas por el noreste de Asia (+6\%) y el sur de Asia (+5\%). Oceanía mostró un crecimiento más moderado en $+3 \%$.

El turismo genera una gran dependencia económica en algunas regiones del mundo. 
Según Acerenza (1984), Lickorish (1994) y

McIntosh, Goeldner y Brent (2001), los impactos positivos del turismo se efectivizan en la creación de empleo directos e indirectos, la generación de divisas, la utilización de la infraestructura y productos locales, el mayor equilibrio en la balanza de pagos, la contribución al Producto Bruto Geográfico y la generación de una mayor diversificación de actividades económicas.

Los efectos ambientales relacionados al calentamiento global ponen en riesgo la actividad turística y la estabilidad económica de varios países producto de la pérdida de atractivos naturales y las modificaciones en el clima, especialmente en aquellas regiones donde existe una marcada dependencia hacia la actividad. Todos los operadores turísticos son sensibles al clima (Scott y Lemieux, 2010). Ello deriva en una incidencia negativa en la economía local. Según el Panel Intergubernamental del Cambio Climático (IPCC, 2007), el cambio climático perjudicará la habilidad de los destinos en conseguir el desarrollo sustentable, debido a que sufrirán serias consecuencias económicas, sociales y ambientales.

\section{Metodología}

La metodología empleada constituye una combinación de distintas perspectivas teóricas que permiten obtener una visión general del estado de la cuestión a nivel mundial y de su manifestación a nivel local. Para responder al objetivo propuesto el trabajo de investigación se dividió en distintas etapas acompañadas de técnicas cualitativas: 1. Revisión bibliográfica y documental, a fin de realizar un correcto análisis del Calentamiento Global, su origen y sus consecuencias. Implicó la lectura de diversas fuentes documentales, entre ellas la UNESCO, el Panel Intergubernamental de Expertos sobre el Cambio Climático (IPCC), la Organización Mundial del Turismo (OMT), la Organización Mundial Meteorológica (OMM) y periodísticas, como National Geographic. 2. Análisis de los principales destinos amenazados teniendo en cuenta la Teoría Social del Riesgo (Cardona, 1993; Wilches-Chaux, 1993) y el binomio clima-turismo (Gómez Martin, 2005). Se especificaron casos emblemáticos cuya selección responde al papel del turismo en el desarrollo económica del lugar o a su riqueza en materia de atractivos turísticos. 3. Elaboración de un esquema donde presentan los principales efectos del calentamiento global, riesgos y amenazas, y las regiones turísticas más vulnerables con base en la regionalización turística de la OMT: Las Américas, Europa, África, Oriente Medio y Asia y el Pacífico.

\section{Resultados}

\section{Efectos del calentamiento global en destinos turísticos}

\subsection{El descongelamiento de las nieves eterna}

El aumento de la temperatura atmosférica en las regiones sub-polares o de gran altitud genera el descongelamiento de las nieves eternas y el retroceso de glaciares. Tal como 
establece la UNESCO (2009), un incremento de temperatura de $4^{\circ} \mathrm{C}$ eliminaría prácticamente todos los glaciares de la Tierra. El volumen de la mayoría de los glaciares de montaña y de otras masas de hielo se ha reducido desde la Pequeña Edad de Hielo, que tuvo lugar entre los siglos XVII y XIX. Sin embargo, en el pasado reciente los glaciares han comenzado a derretirse a velocidades que no pueden ser explicadas por la variabilidad natural del clima (UNESCO, 2009).

Entre los principales efectos se encuentran:

$>$ Pérdida de ecosistemas y afectación en la flora y fauna. Así también, los ecosistemas montañosos corren el peligro de que las especies vegetales y animales cambien de nicho ecológico para adaptarse a los cambios del ambiente.

$>$ Formación de lagos glaciarios, es decir, acumulaciones de agua en una depresión como consecuencia de la erosión ocasionada por un glaciar. Las orillas de tales lagos presentan morenas, acumulaciones de tierra y piedras depositadas por el glaciar (Tarbuck y Lutgens, 2005). Las mismas pueden colapsar al llenarse el lago, provocando repentinas y violentas inundaciones en los valles inferiores. Ello puede tener consecuencias desastrosas para la población y para la biodiversidad de las regiones al pie de los valles.

$>$ Alteraciones en los ciclos hidrológicos de las cuencas alimentadas por glaciares. Con la reducción del hielo, en un principio pueden producirse inundaciones, pero la provisión de agua se verá reducida, favoreciendo las hambrunas y la propagación de enfermedades.

$>$ Disminuye la superficie reflectante de la tierra. Tiene un efecto de retroalimentación positiva, en el sentido que intensifica el calentamiento atmosférico al ser más la energía absorbida por la tierra y menos la reflejada (Albedo).

El PICC-AR5 (2014) menciona que las proyecciones indican que es muy probable que continue la disminución de la capa de hielo marino del Ártico, la capa de nieve primaveral del hemisferio norte $y$ el volumen global de los glaciares. Según el Informe de las Naciones Unidas (ONU, 2006), durante el último siglo, el volumen del hielo marítimo en el Ártico disminuyó un 40\%. La biodiversidad del Ártico está en peligro, se incluyen reducciones en las poblaciones de aves marinas y mamíferos marinos como leones marinos, y la disminución de las cosechas de salmón. El oso polar depende del hielo marino del verano para cazar focas, por lo cual, a medida que la extensión del hielo marino se reduce, también lo hace el hábitat del oso polar. El declive de estos icónicos animales del Ártico afecta a las comunidades originarias, pero también al turismo.

En la región de las Américas, el aumento de la temperatura en Alaska, especialmente en invierno, ha generado que se derrita el suelo permafrost, llamado así a "...cualquier subsuelo permanentemente helado. Se encuentra normalmente en las regiones árticas y subárticas" ... (Tarbuck y Lutgens, 2005, p. 695). La reducción del hielo marino protector y el aumento del nivel del mar hacen que las aldeas costeras sean más 
vulnerables. Entre los efectos de ello se encuentran: el hundimiento de viviendas y edificios, y el agrietamiento o desmoronamiento de cimientos, carreteras y líneas eléctricas construidas sobre el permafrost.

Por otra parte, el Parque Nacional Glacier en Estados Unidos es uno de los afectados por el aumento de la temperatura global, pudiendo desaparecer completamente hacia 2020 (Unión Internacional para la Conservación de la Naturaleza- IUCN, 2017). El informe explica que desde la creación del parque en 1910 se ha observado una drástica disminución del número de glaciares, que han pasado de 150 a 25 .

En Europa, la región montañosa de los Alpes (Austria, Alemania, Francia, Italia, Mónaco, Eslovenia, Suiza) también se ve afectada, pudiendo desaparecer hacia 2050 de continuar el descenso en las nevadas de la región (IUCN, 2017). La región de los Alpes se está calentando tres veces más que la media global. Tal es el caso de JungfrauAletsch-Bietschhorn en Suiza (UNESCO, 2009). Por su parte, en África, según el Informe de las Naciones Unidas (ONU, 2006), durante el último siglo la cobertura de hielo del Monte Kenia perdió 92\% de su masa.

En la región de Asia y el Pacífico, según National Geographic (2019a) el Himalaya es una de las regiones más afectadas. El derretimiento de las nieves eternas generó el desborde del Río Kosi e inundaciones en el 2008, con más de 20 millones de personas obligadas a abandonar sus hogares. Así también, el Parque Nacional de Sagarmatha en Nepal considerado Patrimonio de la
Humanidad (UNESCO, 2009) al pie del monte Everest, es amenazado por el calentamiento global. Este parque alberga especies emblemáticas como la pantera de las nieves y es Patrimonio Mundial desde 1979.

\subsection{Aumento del nivel del mar}

Tal como se menciona en el PICC-AR5 (2014), el cambio del nivel del mar se ha producido desde 1950 a tasas sin precedentes. Los escenarios para el 2100 indican que el nivel global del mar podría aumentar entre un 0.18 y un $0.59 \mathrm{~m}$ (PICC, 2007). La UNESCO (2009) estableció un aumento global promedio del nivel del mar entre $0,1 \mathrm{~m}$ y $0,2 \mathrm{~m}$ durante el siglo $\mathrm{XX}$ debido a la dilatación térmica del agua del océano y al derretimiento de masas de hielo en glaciares y calotas. El retroceso de la línea de costa y la pérdida sedimentaria, constituyen algunos de los indicios del crecimiento del nivel del mar.

Entre los principales efectos ambientales del aumento del nivel del mar se pueden mencionar:

$>\mathrm{El}$ incremento del riesgo a inundaciones en áreas costeras por efectos del crecimiento del nivel del mar.

La pérdida de pantanos y manglares por la intrusión de agua de mar. Los manglares constituyen defensas naturales frente a la erosión, por lo cual, a causa del aumento del nivel del mar, se produce un crecimiento del ritmo erosivo.

Numerosos bienes declarados Patrimonio de la Humanidad por la UNESCO en el mediterráneo europeo, están amenazados por el crecimiento de la erosión 
costera y las inundaciones provocadas por el aumento del nivel del mar. Los principales riesgos para el próximo siglo, deben afrontarlos Venecia (Italia), la Piazza del Duomo en Pisa (Italia) y la ciudad medieval de Rodas (Grecia). El aumento del nivel del mar amenaza con generar inundaciones en 300 kilómetros de costa entre el norte del Adriático y el oeste de Italia, por lo que la ciudad de Venecia es uno de los destinos en mayor riesgo. National Geographic (2018) estableció un crecimiento de la marea de 156 centímetros metros sobre el nivel del mar, para el 2019, en lo que se conoce como acqua alta.

En la región de Oriente Medio, el Parque Nacional de Ichkeul (Túnez), Patrimonio Mundial, es un humedal y escala para aves migratorias. Según la UNESCO (2009), el aumento del nivel del mar, tendrá varios efectos adversos en los humedales costeros incluyendo erosión e inundaciones costeras, la pérdida de hábitats y el incremento de la salinidad de los estuarios. A su vez, las zonas húmedas y las turberas tienen un papel significativo en el ciclo global del carbono, ello significa que de afectarse es posible liberar carbono a la atmosfera y aumentar a un más la temperatura.

En la región Asia y el Pacífico, las islas del Océano Pacífico se encuentran en situación de riesgo (Simon et al., 2016). Tal como explican los autores, las islas Salomón, en mitad del Pacífico occidental, son una de las más afectadas. El archipiélago, formado por unas 1.000 islas que juntas apenas superan los $28.000 \mathrm{Km}^{2}$ de extensión, es el hogar de más de medio millón de personas. De origen volcánico, muchas se encuentran casi al nivel del mar, por lo cual, cinco pequeñas islas de las Salomón han desaparecido. Simon et al., (2016) también explican que otras seis islas han perdido hasta el $62 \%$ de su tierra y, además, el ritmo de avance del mar se está acelerando. Hasta los años 60, las aguas arrebataban apenas un $0,1 \%$ por unidad de área, el porcentaje se elevó al 0,5\% anual para el 2002 llegando a 1,9\%. En la isla de Nuatambu, habitada por 25 familias, el mar avanzó sobre la mitad de la tierra de la isla, destruyendo 11 casas. Como consecuencia, la población ha optado por mudarse a las zonas más elevadas o cambiar de isla.

Por su parte, el archipiélago de las Islas Maldivas, ubicado en el Océano Índico, al suroeste de Sri Lanka e India, está formado por cerca de 1200 islas amenazadas por el aumento medio del nivel del mar. Ello obligó a desalojar a los residentes de 16 de las islas. Si continúa el actual ritmo se cree que en 100 años las Maldivas quedarán completamente sumergidas (IUCN, 2017). Finalmente, los manglares de Sundarbans (India- Bangladesh) constituyen el área manglar más grande del mundo (más de $10.000 \mathrm{~km}^{2}$ de tierra y agua). Se ubican entre el delta de los ríos Ganges, Brahmaputra y Meghna en la bahía de Bengala. Tal como menciona la UNESCO (2009) está atravesado por una compleja red de agua, estanques y pequeñas islas con bosques de mangles adaptados al agua salada. Los manglares funcionan como protecciones naturales contra ciclones tropicales y como filtro entre les aguas dulces y saladas, además de constituir un ambiente ideal para el crecimiento de 
especies de peces e invertebrados marinos. Además del aumento global del nivel del mar los Sundarbans sufren una subsidencia natural (Sanyal, 2002), lo cual provoca un aumento del nivel del mar total de $3,1 \mathrm{~mm}$ por año.

\subsection{Aumento de la temperatura del agua} El aumento de la temperatura del agua puede acarrear varios efectos ambientales, entre ellos:

$>$ Los ecosistemas marinos pueden verse afectados por los cambios en la temperatura del agua marina. Esto incluye modificación en los comportamientos migratorios, composición de las comunidades y funcionamiento de los ecosistemas.

> Intensificación de la velocidad del viento de los ciclones tropicales asociado a la formación de bajas presiones. Según un estudio del IPCC, a nivel global en las proyecciones el número total de los ciclones tropicales disminuirá, mientras que el número de ciclones de intensidad 5 (o más) aumentará.

La Gran Barrera de Coral de Australia (Patrimonio de la Humanidad) es uno de los ecosistemas marinos más afectados por el calentamiento global y los cambios de temperatura en las corrientes marinas. En este ecosistema habitan cerca de 400 especies de coral y más de mil 500 de peces. La belleza paisajística permite que el turismo tenga un aporte significativo a la economía australiana.

National Geographic (2019b) estableció que más de dos tercios del coral de la Gran Barrera de Coral de Australia ha sufrido graves procesos de blanqueamiento en menos de un año. Durante el 2016 y 2017 , estas decoloraciones devastaron cerca de mil $500 \mathrm{~km}$ de coral producto de una fuerte ola de calor. La IUCN (2017) explica que cuando las algas que dan coloración a los corales mueren debido al aumento de la temperatura del agua, los corales adquieren color blanco. Producto de que los procesos de blanqueamiento son más frecuentes y la oportunidad de recuperarse es reducida, la Gran Barrera de Coral se encuentra bajo un Plan de Sostenibilidad con metas hacia 2050 que permiten conservar este importante Patrimonio Natural de la Humanidad.

Por su parte, los llamados ciclones tropicales o tifones, se originan normalmente en las regiones tropicales donde el agua del océano tiene una temperatura de $26^{\circ} \mathrm{C}$ en adelante. $\mathrm{El}$ aire más caliente asciende por una disminución densidad, alejándose de la superficie del océano. Este fenómeno causa una disminución de la presión del aire cerca del océano. El aire en ascenso se enfría y condensa formando nubes que giran por el Efecto de Coriolis. Los ciclones alcanzan una velocidad mínima $74 \mathrm{mph}$ y ad se pueden dividir en 5 categorías según la escala Saffir Simpson, donde la categoría 1 es la menos impactante por menor velocidad y la 5 la más peligrosa y catastrófica.

La región de las Américas es una de las más vulnerables a los huracanes. Moore (2011), realiza un análisis macroeconómico del impacto del cambio climático en países del Caribe, y detalla que un deterioro de las condiciones climáticas tendrá un impacto directo en el crecimiento económico. De 
esta forma, el consumo se ve afectado negativamente por el cambio climático, lo mismo sucede con la inversión. En este sentido, el Caribe perdió más de US\$1,000 millones en ingresos por turismo (Organización de Turismo del Caribe) ${ }^{1}$ después de que huracanes afectaran la imagen turística y disminuyera el número de visitantes.

En septiembre de 2017, los huracanes Irma y María provocaron grandes efectos en Puerto Rico, las Islas Vírgenes y otros países caribeños. Incluyó daños económicos, destrucción de viviendas refugiados, destrucción del tendido eléctrico e infraestructura de comunicaciones. El turismo se vio afectado incluso en destinos cuyas playas y complejos turísticos no se encontraban en áreas de riesgo. Ello se debió a la gran cobertura mediática que contribuyó a una percepción negativa de los destinos.

El turismo en Cuba, uno de los sectores más importantes para la economía del país, también se vio afectado por Irma. Los huracanes provocaron una disminución de visitantes. Por su parte, durante la temporada de huracanes en México, se intenta promover ofertas turísticas que amortigüen la vulnerabilidad de los destinos.

\subsection{Cambios en las corrientes marinas}

Las corrientes marinas pueden ser superficiales o profundas. Las primeras deben su movimiento al viento y están influenciadas por la distribución de los continentes y la rotación terrestre. El Efecto Coriolis desvía los vientos en el hemisferio norte de forma circular en sentido de las agujas del reloj y en el hemisferio sur en sentido contrario. Por su parte, las corrientes profundas o Termohalinas, son producidas por el hundimiento del agua producto de una alta densidad, asociada a la baja temperatura o alta salinidad. El movimiento de la Corriente Termohalina comienza en el Océano Atlántico cuando se genera una corriente de agua fría muy salina, la Corriente Ártica, que se hunde y dirige hacia el sur. A $60^{\circ}$ latitud sur, la corriente asciende al ser empujada por otra corriente aún más fría, la Corriente Antártica, que fluye hacia el norte por el océano Atlántico, Índico y Pacífico.

El calentamiento global puede acarrear un debilitamiento de la corriente profunda por aumento de la temperatura del agua y reducción de la salinidad, derivado del derretimiento de glaciares. La región de Europa, es una de las más amenazadas por este fenómeno. El clima de Europa Nórdica está regulado en gran parte por la Corriente Marina del Golfo, una corriente cálida proveniente del Golfo de México que tiene su origen en la circulación profunda. Su presencia amortigua las temperaturas frías típicas de una alta latitud. Se corroboró una ralentización de la Corriente Marina del Golfo (IPCC, 1995) debido a mayores precipitaciones en el Océano Atlántico y el derretimiento del hielo del Ártico. Ello

\footnotetext{
${ }^{1}$ Consultado el 1 de diciembre de 2018 (https://www.ecured.cu/Organizaci\%C3\%B3n_de_ Turismo_del_Caribe).
} 
genera una disminución de la concentración de sal, por lo cual, se reduce su densidad del agua y se hunde más lentamente. Así también, el PICC-AR5 (2014) menciona que las proyecciones indican que los océanos continuarán calentándose y el calor se extenderá al océano profundo, afectando los patrones de circulación.

\subsection{Crecimiento de precipitaciones}

El calentamiento atmosférico genera mayores precipitaciones convectivas en algunas regiones. Las precipitaciones globales podrán aumentar cerca del 2\% (IPCC, 1995). La Administración Nacional de la Aeronáutica y del Espacio (NASA) ${ }^{2}$ estableció que los años 2016 y 2017 fueron los más calientes de la Tierra de los registrados desde 1880 y ello produce desequilibrios climáticos en diferentes lugares, lo que resulta en fenómenos extremos como inundaciones. Tal como establece el IPCC (1995), la tierra sufrirá un calentamiento mayor que los océanos y las áreas del norte serán más afectadas.

La región de la OMT más amenazada por aumento de precipitaciones es Las Américas. Entre 1900 y 2005, se dio un aumento significativo en la cantidad de lluvias en muchas regiones, principalmente en la parte este de América del Norte y América del Sur. Sin embargo, la frecuencia de las precipitaciones extremas aumentó en gran parte de las áreas terrestres (IPCC, 1990), incluyendo la zona ecuatorial.

\footnotetext{
${ }^{2}$ Consultado el 1 de diciembre de 2018

(https://www.nasa.gov/).
}

La IUCN (2017) menciona que Machu Picchu (Perú), ubicada en la cima de una de las montañas que componen la Cordillera de los Andes peruanos, ha registrado importantes acumulados de precipitación a lo largo de los últimos años. Las lluvias torrenciales han causado grandes deslizamientos en las laderas de las montañas convirtiendo el trayecto hasta Machu Picchu en una travesía muy peligrosa. Además, en el año 2010 una grave inundación en el pueblo de Aguas Calientes dejó aislados a cerca de 2000 turistas.

En Argentina, las precipitaciones en el noroeste (NOA) siguen rompiendo récords, según el Servicio Meteorológico Nacional en los 10 primeros días de mayo, llovió en Salta cinco veces más de lo esperado (41.4 $\mathrm{mm}$ cuando el promedio es $7.8 \mathrm{~mm}$ ). Los efectos de estas abundantes precipitaciones incluyen solo los conocidos anegamientos en las ciudades y pueblos, sino también son desencadenantes de episodios impredecibles.

\subsection{Sequias más intensas y olas de calor}

Las sequias amenazan varias regiones del planeta $y$ son intensificadas por el calentamiento global y la consecuente disminución de las precipitaciones en algunas regiones. El IPCC (1990) explica que es probable que existan sequías más largas e intensas a partir de 1970, especialmente en los trópicos y los subtrópicos. El aumento del clima seco, 
junto con las temperaturas más elevadas y una reducción de las lluvias, contribuirán a generar cambios en diversas regiones.

Los principales efectos de ello son:

$>$ Avance de la desertificación: se produce donde el agua en general es escasa o su abastecimiento es irregular.

$>$ Efectos en ecosistemas: están asociados principalmente a la pérdida de especies faunísticas y florísticas por la disminución de precipitaciones.

$>$ Olas de calor: las sequías tienden a ser cada vez más cálidas, produciendo en algunas regiones el alcance de temperaturas extremas.

Incendios: ligado al aumento de temperatura y a la escasez de agua, aumentan de forma inevitable las sequías y los incendios forestales, amenazando la biodiversidad

La OMT (2007) explica que el avance de la desertificación se produce especialmente en la región de África subsahariana. En 2009, Etiopia y Kenia atravesaron la peor crisis alimentaria del último cuarto de siglo por una ausencia de precipitaciones (Organización de las Naciones Unidas para la Alimentación y la Agricultura- FAO, 2005). Tal como presenta la FAO (2005), Ouargla, en el desierto del Sahara de Argelia, registró una temperatura máxima de $51,3^{\circ} \mathrm{C}$, siendo la temperatura histórica más alta registrada en Argelia. La ciudad de Kebili, en Túnez, mantiene el récord de temperatura más alta registrada en África con $55^{\circ} \mathrm{C}$.

El lago Nakuru de Kenya, al que arriban gran cantidad de turistas para contemplar poblaciones de aves, atraviesa una insuficiente de agua. $\mathrm{Al}$ avanzar el desierto también retrocede el hábitat de la fauna y la flora silvestres. En Tanzanía, se estima que las nieves del Kilimanjaro habrán desaparecido por completo para el año 2020. Se ha registrado una disminución del número de leones, elefantes y rinocerontes en África, que hace más difícil el turismo de safari. Algunas especies de animales y plantas podrían sufrir riesgo de extinción.

Por otra parte, en la región de Europa, el aumento de temperatura es notorio en la región del mediterráneo. El IPCC (2006) pone el foco en la cuenca mediterránea, que ya ha aumentado su temperatura en $1,4^{\circ} \mathrm{C}$, lo que supone casi medio grado más que la media global. Según National Geographic (2019c), las olas de calor son cada vez más frecuentes en España, a su vez, es mayor el riesgo de incendios. En el 2015 una ola de calor afectó España con 26 días de duración. En la región de Las Américas, la estación de Furnace Creek del Parque Nacional del Valle de la Muerte en el estado de California (EE. UU) registró una temperatura de $52^{\circ} \mathrm{C}$. La estación tiene el récord de la temperatura más alta registrada en la Tierra con $56,7^{\circ} \mathrm{C}$ en 1913. Así también, el centro de Los Ángeles estableció un mínimo mensual en julio de $26,1^{\circ} \mathrm{C}$ por la noche; y Chino 
(California) vio una temperatura récord de 48, $9^{\circ} \mathrm{C}$ (OMM, 2018) ${ }^{3}$.

Según la Organización Mundial de la Salud $^{4}$, la exposición excesiva al calor tiene una amplia variedad de efectos fisiológicos en los seres humanos que pueden resultar en muertes prematuras e incapacidad. La excesiva exposición al calor durante periodos prolongados del día y la noche puede producir insuficiencias cardiovasculares, respiratorias y renales, así como diabetes. Los grupos de población más vulnerables son las personas mayores, los niños, las mujeres embarazadas, entre otros.

Finalmente, en la región de Asia y el Pacífico, se destaca Australia como un país en riesgo por el crecimiento de sequias y episodios de olas de calor. Australia ha experimentado un incremento de la temperatura promedio de $0.7^{\circ} \mathrm{C}$ entre 1910 y 1999 del cual la mayor parte ha ocurrido desde 1950 (Plummer, Lin y Torok, 1995). En este sentido, Sídney soportó la temperatura más cálida en 80 años durante enero del 2018 con $47,3^{\circ} \mathrm{C}$, medio grado debajo del récord histórico de $47,8^{\circ} \mathrm{C}$ en 1939.

El aumento de la temperatura trae aparejado un crecimiento de incendios en Australia Meridional, Victoria y Australia Occidental. Así también, en la región Nueva Gales del Sur la llamada Región de las Montañas Azules (Patrimonio de la Humanidad) donde los bosques templados de eucaliptos se hallan entre los ecosistemas más

\footnotetext{
${ }^{3}$ Consultado el 1 de diciembre de 2018

(https://public.wmo.int/es).
}

dependientes del fuego del mundo. Tal como menciona la UNESCO (2009), si el intervalo entre incendios forestales intensos cambia de ciclos largos de diez o veinte años a menos de seis, habría una disminución significativa en la diversidad de las principales especies de eucaliptos y de otra flora, con grandes amenazas en su valor como Patrimonio Mundial y turístico.

\section{Discusión}

La Tabla 1 y Figura 1 sintetizan los principales efectos ambientales del calentamiento global (retroceso de glaciares, aumento del nivel del mar, aumento de la temperatura oceánica, cambios en las corrientes marinas, crecimiento de las precipitaciones, seguías más intensas) y algunos de los destinos más afectados dentro de las regiones turísticas planteados por la OMT. La región Asia y el Pacifico, es una de las más vulnerable, el crecimiento del nivel del mar pone en riesgo la supervivencia de algunos destinos turísticos insulares como Maldivas y ecosistemas como los Manglares de Sundarbans en India-Bangladesh. A su vez, el aumento de la temperatura oceánica lleva a la formación de huracanes más intensos en el sudeste asiático y a efectos en los ecosistemas como el blanqueamiento de la Gran Barrera Coralina, uno de los principales atractivos turísticos naturales de Australia. Por otra parte, el calentamiento global acarrea el derretimiento de las nieves eternas en algunos destinos montañosos,

\footnotetext{
${ }^{4}$ Consultado el 1 de diciembre de 2018 (https://www.who.int/es).
} 
tales como el Parque Nacional de Sagarmatha en Nepal o la generación de incendios en gran parte de Australia como la región turística Nueva Gales del Sur.

Por su parte, Europa es otra de las regiones más afectadas por el calentamiento global, se destaca el debilitamiento de la corriente superficial del Golfo que lleva a una disminución de la temperatura atmosférica superficial en destinos bañados por el Océano Atlántico. Así también, existe riesgo de retroceso de glaciares en los Alpes, atractivo turístico natural que permite el desarrollo de diversas actividades turísticas y/o recreativas como ski. Por otra parte, se destacan, el crecimiento de olas de calor en toda Europa, con mayor afectación en la región mediterránea, ello incluye destinos como Girona en España; y la amenaza del aumento del nivel del mar con el riesgo de inundaciones en algunos destinos turísticos como Venecia en Italia. En el caso de Las América, especialmente en la región de América Central o el Caribe, existe también el riesgo de formación de huracanes más intensos, derivado de un calentamiento de la temperatura oceánica. En América del Norte se evidencian situaciones de riesgo por derretimiento de nieves eternas como es el caso del Parque Nacional de los Glaciares en EE. UU.; o por aumento de sequias extremas, amenaza principal de algunos destinos como Los Ángeles en EE UU. Finalmente, en Las Américas también se observa un crecimiento de precipitaciones, principalmente en destinos de América del Sur como Machu Pichu en Perú.

En el caso de la región de África, una de las principales amenazas es el retroceso de glaciares del Monte Kenia, un atractivo turístico natural de importancia; y el avance de la desertificación en la región del Sahara. En el caso de la región turística de Oriente Medio, existen riesgos asociados al aumento del nivel del mar, tal como es el caso del Parque Nacional de Ichkeul en Túnez.

Tabla 1. Efectos ambientales del calentamiento global y regiones turísticas en riesgo

\begin{tabular}{|c|c|c|}
\hline \multicolumn{3}{|c|}{ Efectos ambientales del calentamiento global y regiones turísticas en riesgo } \\
\hline \multicolumn{2}{|c|}{ Amenazas ambientales } & Regiones de la OMT en riesgo \\
\hline \multirow[t]{3}{*}{$\begin{array}{l}\text { Retroceso de } \\
\text { glaciares }\end{array}$} & $\begin{array}{l}\text { Pérdida de } \\
\text { ecosistemas }\end{array}$ & \multirow{3}{*}{$\begin{array}{ll} & \text { LAS AMÉRICAS: } \\
& \text { Destinos de Alaska (Estados Unidos) } \\
> & \text { Parque Nacional Glacier (Estados Unidos) } \\
& \text { EUROPA: } \\
& \text { Destinos de los Alpes como Jungfrau-Aletsch- } \\
\text { Bietschhorn (Suiza) } & \\
\text { ÁFRICA: }\end{array}$} \\
\hline & Avalanchas & \\
\hline & Inundaciones & \\
\hline
\end{tabular}




\begin{tabular}{|c|c|c|}
\hline & & $\begin{array}{l}\text { Monte Kenia (Kenia) } \\
\text { ASIA Y EL PACÍFICO: } \\
\text { Destinos del Himalaya como el Parque Nacional } \\
\text { de Sagarmatha (Nepal) }\end{array}$ \\
\hline \multirow[t]{2}{*}{$\begin{array}{l}\text { Aumento del } \\
\text { nivel del mar }\end{array}$} & Inundaciones & $\begin{array}{ll}\text { EUROPA } \\
>\begin{array}{l}\text { Destinos bañados por el Mar Mediterráneo como } \\
\text { Venecia (Italia) }\end{array} \\
\text { ORIENTE MEDIO: } \\
>\quad \text { Parque Nacional de Ichkeul (Túnez) } \\
\\
\text { ASIA Y EL PACÍFICO: } \\
>\text { Parque Nacional de Komodo (Indonesia) } \\
> & \text { Islas Salomón } \\
& \text { Islas Maldivas }\end{array}$ \\
\hline & $\begin{array}{l}\text { Pérdida de } \\
\text { manglares y } \\
\text { humedales } \\
\text { costeros }\end{array}$ & $\begin{array}{ll} & \text { ORIENTE MEDIO: } \\
>\quad \text { Parque Nacional de Ichkeul (Túnez) } \\
\\
\text { ASIA Y EL PACÍFICO: } \\
>\quad \text { Manglares de Sundarbans (India- Bangladesh) }\end{array}$ \\
\hline \multirow{2}{*}{$\begin{array}{l}\text { Aumento } \\
\text { temperatura } \\
\text { oceánica }\end{array}$} & $\begin{array}{l}\text { Efectos en } \\
\text { ecosistemas }\end{array}$ & $\begin{array}{ll} & \text { ASIA Y EL PACÍFICO: } \\
>\quad \text { Gran Barrera de Coral (Australia) }\end{array}$ \\
\hline & $\begin{array}{l}\text { Huracanes } \\
\text { más intensos }\end{array}$ & $\begin{array}{ll} & \text { LAS AMÉRICAS: } \\
> & \text { Destinos bañados por el Mar Caribe } \\
> & \text { Destinos de México } \\
> & \text { Miami (Estados Unidos) } \\
& \text { ASIA Y EL PACIFICO } \\
> & \text { Destinos del Sudeste Asiático }\end{array}$ \\
\hline $\begin{array}{l}\text { Modificación } \\
\text { de corrientes } \\
\text { marinas }\end{array}$ & $\begin{array}{l}\text { Debilitamient } \\
\text { o corriente } \\
\text { Termohalina }\end{array}$ & $\begin{array}{ll} & \text { EUROPA: } \\
& \text { Destinos bañados por el Océano Atlántico }\end{array}$ \\
\hline $\begin{array}{l}\text { Crecimiento de } \\
\text { precipitaciones }\end{array}$ & Inundaciones & $\begin{array}{ll}\text { LAS AMÉRICAS: } \\
>\text { Destinos de América del Norte } \\
>\text { Destinos de América Central y América del Sur } \\
\text { como Machu Pichu (Perú) o NOA (Argentina) }\end{array}$ \\
\hline $\begin{array}{l}\text { Sequias más } \\
\text { intensas }\end{array}$ & $\begin{array}{l}\text { Avance de la } \\
\text { desertificació }\end{array}$ & $\begin{array}{ll} & \text { LAS AMÉRICAS: } \\
& \text { Parque Nacional del Valle de la Muerte (EE. UU) }\end{array}$ \\
\hline
\end{tabular}




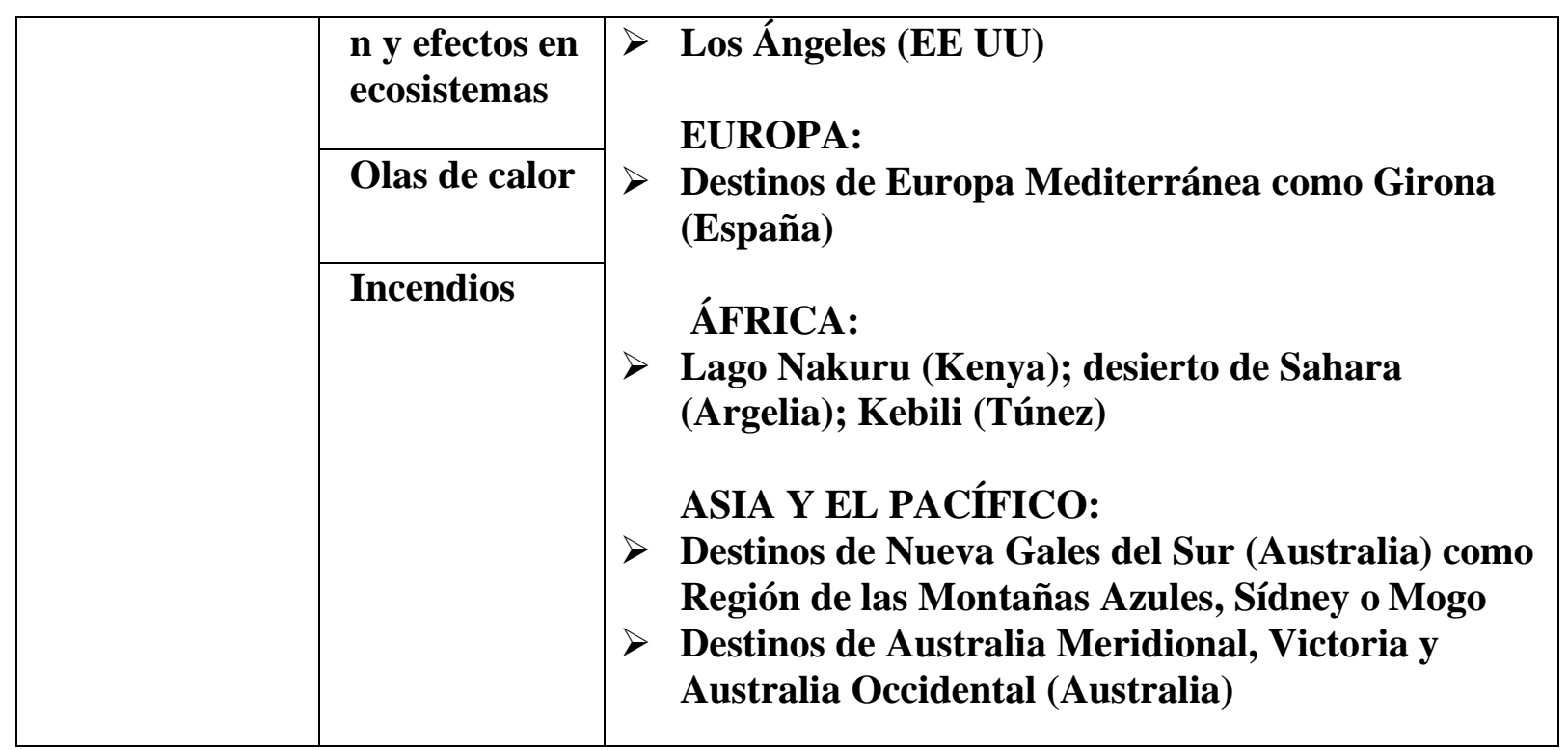

Fuente: elaboración personal (2020)

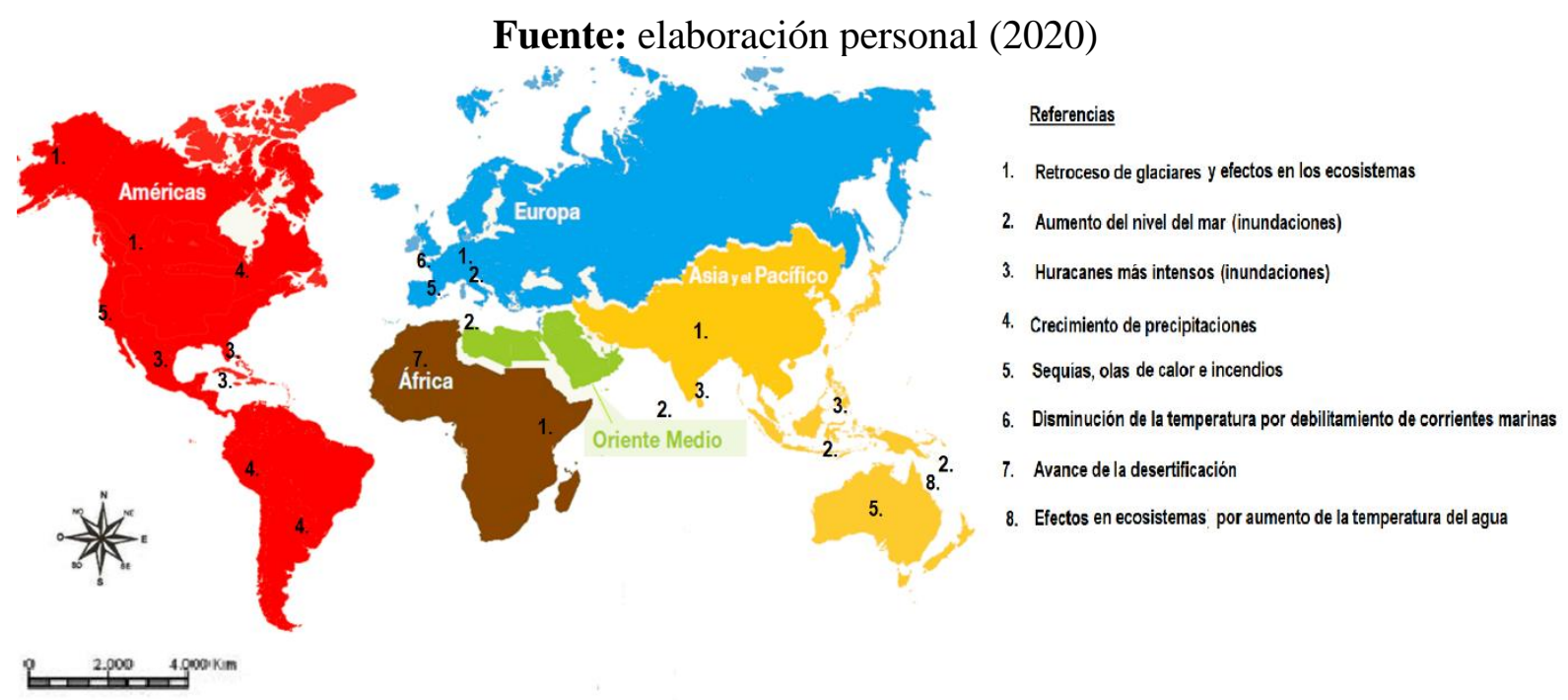

Figura 1. Regiones de la OMT y principales amenazas ambientales del calentamiento global

\section{Conclusiones}

Los efectos del calentamiento global incluyen el derretimiento de las nieves eternas, el aumento del nivel del mar, los cambios en las corrientes marinas y los fenómenos meteorológicos extremos (huracanes, inundaciones, precipitaciones, sequias, olas de calor). Estos generan situaciones de riesgo en todo el globo y fueron planteados para todas las regiones de la OMT. Se destaca Asía 
y el Pacifico como una de las más amenazadas por el aumento del nivel del mar, suceso que pone en riesgo la supervivencia de algunas islas y destruye los ecosistemas; o el aumento de la temperatura oceánica que amenaza con la formación de huracanes más intensos. Así también, se observaron otros fenómenos meteorológicos extremos como sequias en muchos destinos turísticos.

Estas situaciones afectan al turismo directa e indirectamente de distintas formas. Desde el punto de vista ambiental, abordado en este artículo, modifican situaciones meteorológicas recurrentes que favorecen a muchos destinos, especialmente a aquellos de sol y playa; generan un mayor riesgo de desastres naturales; y amenazan la supervivencia de atractivos naturales. De estas cuestiones se derivan otras de índole más social o económicas como el crecimiento de la vulnerabilidad de los turistas en relación a su salud y seguridad; riesgo de destrucción de la infraestructura básica del lugar y pérdidas económicas.

Los destinos turísticos deben reducir su vulnerabilidad física frente a los fenómenos peligrosos que acarrea el calentamiento globo, ello requiere conocer los principales riesgos ambientales. Sin embargo, es necesario poner en reflexión que la alteración de los patrones meteorológicos podría ofrecer, en algunas regiones, oportunidades al sector turístico basadas en el surgimiento de nuevas modalidades turísticas. De esta forma, en las regiones montañosas afectadas por el derretimiento de las nieves eternas, donde el de turismo de ski es una práctica recurrente, el desarrollo del turismo de naturaleza y actividades recreativas como tracking, rafting, escalada, entre otros, permite pensar en nuevas oportunidades en temporada baja, permitiendo también superar los problemas de la estacionalidad.

\section{Referencias bibliográficas}

Acerenza, M. (1984). Administración del Turismo. Conceptualización y Organización. (volumen 1). México: Editorial Trillas.

Amelung B., y Viner D. (2006). La sostenibilidad del turismo en el Mediterráneo: explorando el futuro con el Índice de comodidad del turismo. Diario de Turismo Sostenible, 14(4), 349-366.

Andrade, J. (1998). Turismo. Fundamentos e dimensões. São Paulo: Papirus,

Barros, V. (2003). Cambio Climático. En Barros, Menéndez y Nagy (Eds.), El cambio climático en el Río de La Plata (pp. 3-12). Buenos Aires: Proyecto Assessments of Impacts and Adaptations to Climate Change (AIACC).

Barros, V. (2005). El Cambio Climárico Global. Buenos Aires: Libros del Zorzal.

Barry, R., y Chorley, R. (2003). Atmosphere, Weather, and Climate. New York: Routledge Taylor \& Francis Group.

Bennett, M., y Doyle, P. (1997). Environmental Geology. Geology and Human Environment. Chichester, New York: John Wile \& Sons.

Blaikie, P., Cannon, T., David, I., y Wisner, B. (1996). Vulnerabilidad. El entorno social, político y económico de los 
desastres. Panamá: LA RED de estudios sociales.

Cardona, O. (1993). Evaluación de la amenaza, la vulnerabilidad y el riesgo. En A. Maskrey (Ed.), Los desastres no son naturales (pp. 51-74). Bogotá: LA RED de estudios sociales.

Cuadrat, J. M., y Pita, M. F. (1997). Climatología. (4a ed.). Madrid: Cátedra.

Gómez Martín, B. (2005). Reflexión Geográfica en torno al binomio Clima- Turismo. Boletín de la AGE, (40), 111-134.

Gómez Piñiero, F. (2004). Aportaciones de la geografía al estudio científico del turismo. España: Universidad de Deusto.

Hamilton, J., y Lau, M. (2004). The role of climate information in tourist destination choice decision-making (Working Paper FNU-56, Hamburg University and Centre for Marine and Atmospheric Science, Hamburg). En S. Gössling y C. M. Hall (Eds.), Tourism and global environmental change (p. 35). Londres: Routledge.

Houghton, J., Ding, D., Griggs, J., Noguer, M., Van Der Linden, P., y Xiaosu, Y. (2001). Climate Change 2001: The Scientific Basis: Contributions of Working Group I to the Third Assessment Report of the Intergovernmental Panel on Climate Change. Cambridge University Press.

Intergovernmental Panel on Climate Change (IPCC). (1990). I Assessment Report
(AR1) - Physical Science Basis. Cambridge.

Intergovernmental Panel on Climate Change (IPCC). (1995). II Assessment Report (AR2) - Impacts, adaptation and vulnerability. Geneva.

Intergovernmental Panel on Climate Change (IPCC). (2006). Guidelines for National Greenhouse Gas Inventories (Prepared by the National Greenhouse Gas Inventories Programme). H.S. Eggleston, L. Buendia, K. Miwa, T. Ngara y K. Tanabe (Eds). Japón: IGES.

Intergovernmental Panel on Climate Change (IPCC). (2007). IV Assessment Report (AR4) - Synthesis report. Valencia.

Intergovernmental Panel on Climate Change (IPCC). (2014). Quinto Informe de Evaluación (AR5). Quito, Ecuador: Organización Meteorológica Mundial (OMM) y Programa de las Naciones Unidas para el Medio Ambiente (PNUMA).

Lickorish, L. (1994). Desarrollo de Destinos Turísticos. Políticas y perspectivas. México: Editorial Diana.

Mcintosh, R., Goeldner, C., y Brent R. (2001). Turismo, Planeación, Administración y Perspectivas. Editorial Limusa.

Mieczkowski, Z. (1985), The tourism climatic index: A method of evaluating world climates for tourism. Canadian Geographer / Le Géographe canadien, 29: 220-233. 
doi:10.1111/j.1541-

0064.1985.tb00365.x

National Geographic. (2018). Venecia hasta el cuello. Consultado el 30 de octubre de

2018

https://www.nationalgeographic.com. es/fotografia/foto-del-dia/veneciahasta-cuello_13398.

National Geographic. (2019a). El cambio climático tiene drásticos efectos en la región del Himalaya. Consultado el 5 de febrero de 2019. https://www.nationalgeographicla.co $\mathrm{m} /$ medio-ambiente/2019/02/elcambio-climatico-tiene-drasticosefectos-en-la-region-del-himalaya.

National Geographic. (2019b). La Gran Barrera de Coral agoniza por el cambio climático. Consultado el 1 de abril de 2019. https://www.ngenespanol.com/natura leza/gran-barrera-de-coral-deaustralia-calentamiento-global/

National Geographic. (2019c). España, cada vez más vulnerable al cambio climático. Consultado el 11 de marzo de 2019.

Organización de las Naciones Unidas. (ONU). (2006). Climate change an overview. Conferência das Nações Unidas sobre Mudança Climática. Nairóbi: Relatório do Departamento de Assuntos Econômicos e Sociais.

Organización de las Naciones Unidas para la Alimentación y la Agricultura (FAO). (2005). El estado mundial de la agricultura y la alimentación. Roma: FAO.
Organización Mundial del Turismo (OMT). (2007). Cambio climático y turismo. Responder a los retos mundiales. Madrid.

Organización Mundial del Turismo (OMT). (2018). Panorama del Turisnmo Internacional. UNWTO World Tourism Organization.

Padilla, N. (2013). Respuestas sociales ante riesgos asociados a sudestadas en Mar del Sud, Argentina. En Gestores costeros. De la teoría a la práctica: una aplicación en áreas litorales (pp. 81-93). Mar del Plata: Universidad Nacional de Mar del Plata.

Plummer, N., Lin, Z., y Torok, S. (1995). Trends in the Diurnal Temperature Range Over Australia Since 1951. Atmospheric Research, (37), 79-86.

Sanyal, P. (2002). Sea-Level Rise and Sundarban Mangrove. En G. Quadros (Ed.), Proceedings of the National Seminar on Creeks, Estuaries and Mangroves - Pollution and Conservation (pp. 47-50). India: Thane.

Scott, D., y Lemieux, C. (2010). Weather and climate information for tourism. Procedia Environmental Sciences, (1), 146-183.

Simon, A., Leon, J., Grinham, A., Church, J., Badin, G., y Woodroffe, C. (2016) Interactionsbetween sea-levelrise and wave exposure on reefisl and dynamics in the Solomon Islands. Environ. Res. Lett. 11. IOP Publishing. 
Strahler, A., y Strahler, A. (2000). Geografía Física. Barcelona: Omega.

Tarbuck, E., y Lutgens, F. (2005). Ciencias de la Tierra. Una introducción a la Geografía Física. $8^{\circ}$ edición. Madrid: Pearson Educación S. A.

UNESCO. (2009). Cambio climáticoy Patrimonio Mundial. Estudios de caso. Centro del Patrimonio Mundial de la UNESCO.
Unión Internacional para la Conservación de la Naturaleza (IUCN). (2017). Informe Anual 2017. Glándula, Suiza: UICN.

Wilches-Chaux, G. (1993). La vulnerabilidad global. En A. Maskrey (Ed.), Los desastres no son naturales (pp. 1144). Bogotá: LA RED de estudios sociales. 